\title{
Estrogen receptors are linked to angiotensin-converting enzyme 2 (ACE2), ADAM metallopeptidase domain 17 (ADAM-17), and transmembrane protease serine 2 (TMPRSS2) expression in the human atrium: insights into COVID-19
}

\author{
Hao Wang ${ }^{1,2} \cdot$ Xuming Sun $^{1} \cdot$ Jessica L. VonCannon ${ }^{3} \cdot$ Neal D. Kon ${ }^{4} \cdot$ Carlos M. Ferrario ${ }^{3,5} \cdot$ Leanne Groban $^{1,2}$
}

Received: 14 December 2020 / Revised: 4 January 2021 / Accepted: 6 January 2021 / Published online: 3 February 2021

(c) The Japanese Society of Hypertension 2021

Premenopausal women have a reduced incidence of cardiovascular disease (CVD) compared to postmenopausal women or age-matched men, suggesting a cardioprotective role for estrogen [1]. Although estrogen replacement maintains cardiac structure and function in ovariectomized rodent models, clinical trials of estrogen-based hormone therapy have yielded inconsistent results with regard to improving heart function in older women. Overall, it is critical to further elucidate the functional roles of estrogen, especially its individual receptors, in the heart to develop more effective and specific hormone therapy for postmenopausal women.

Estrogen interacts with the renin-angiotensin system (RAS), one of the most critical pathways in CVD, by inhibiting or downregulating renin, angiotensin-converting enzyme (ACE), and angiotensin II (Ang II) type 1 receptor $\left(\mathrm{AT}_{1}-\mathrm{R}\right)$. However, the effects on cardiac ACE2 expression involve both increases and decreases depending on the species and experimental model studied. The identification of the ACE2 enzyme receptor, which acts with host transmembrane serine protease 2 TMPRSS2 [2], as the primary

$\triangle$ Hao Wang

haowang@wakehealth.edu

1 Department of Anesthesiology of Wake Forest School of Medicine, Winston Salem, NC, USA

2 Department of Internal Medicine-Section of Molecular Medicine of Wake Forest School of Medicine, Winston Salem, NC, USA

3 Department of General Surgery of Wake Forest School of Medicine, Winston Salem, NC, USA

4 Department of Cardiothoracic Surgery of Wake Forest School of Medicine, Winston Salem, NC, USA

5 Department of Physiology \& Pharmacology, Wake Forest School of Medicine, Winston Salem, NC, USA means of cellular entry by the novel $\beta$-coronavirus severe acute respiratory syndrome coronavirus 2 (SARS-CoV-2) justifies the importance of examining the potential contributory function of sex hormones in COVID-19 pathogenesis [3, 4].

Newer lessons from cancer biology have uncovered a critical role of estrogen receptor alpha (ER $\alpha)$ and $\operatorname{ER} \beta$ in regulating oncogenic TMPRSS2-ERG fusion [5]. Both ER $\alpha$ and the recently identified $G$ protein-coupled estrogen receptor (GPER) are expressed in the heart of both humans and rodents, without sex differences [6]. To determine links among estrogen receptors, ACE2 expression, the proteincoding gene ADAM Metallopeptidase Domain 17 [4], and TMPRSS2 [4] in the human heart, we analyzed expression levels of these genes by real-time PCR in the right atrial appendage tissue of patients undergoing heart surgery for the correction of left heart valvular disease, resistant atrial fibrillation or ischemic heart disease $(n=34$, mean age 65 \pm 7 years of age, $79 \%$ men). According to our results, right atrial appendage ACE2 mRNA correlated positively with $\mathrm{ER} \alpha(p=0.004$, Fig. 1A) but not GPER (Fig. 1D) or ER $\beta$ (data not shown) expression. This result is consistent with the findings that estrogen increases ACE2 gene expression in the human atrium, which is inhibited by an antagonist of ER $\alpha$ but not ER $\beta$ [7].

As a key counterregulator of the RAS, ACE2 has beneficial effects in the cardiovascular system by converting Ang II to angiotensin-(1-7), which protects the heart against various stresses through its Mas receptor. RAS inhibitors augment ACE2 levels to protect organs from Ang II overload, which may benefit COVID-19 patients with CVDs [8]. Moreover, the positive correlation between ER $\alpha$ and ACE2 mRNA in human atrial tissue might explain the sex differences observed in the clinical course and morbidity of COVID-19. However, as ACE2 also serves as the main route of entry for SARS-CoV-2 into human host cells, 

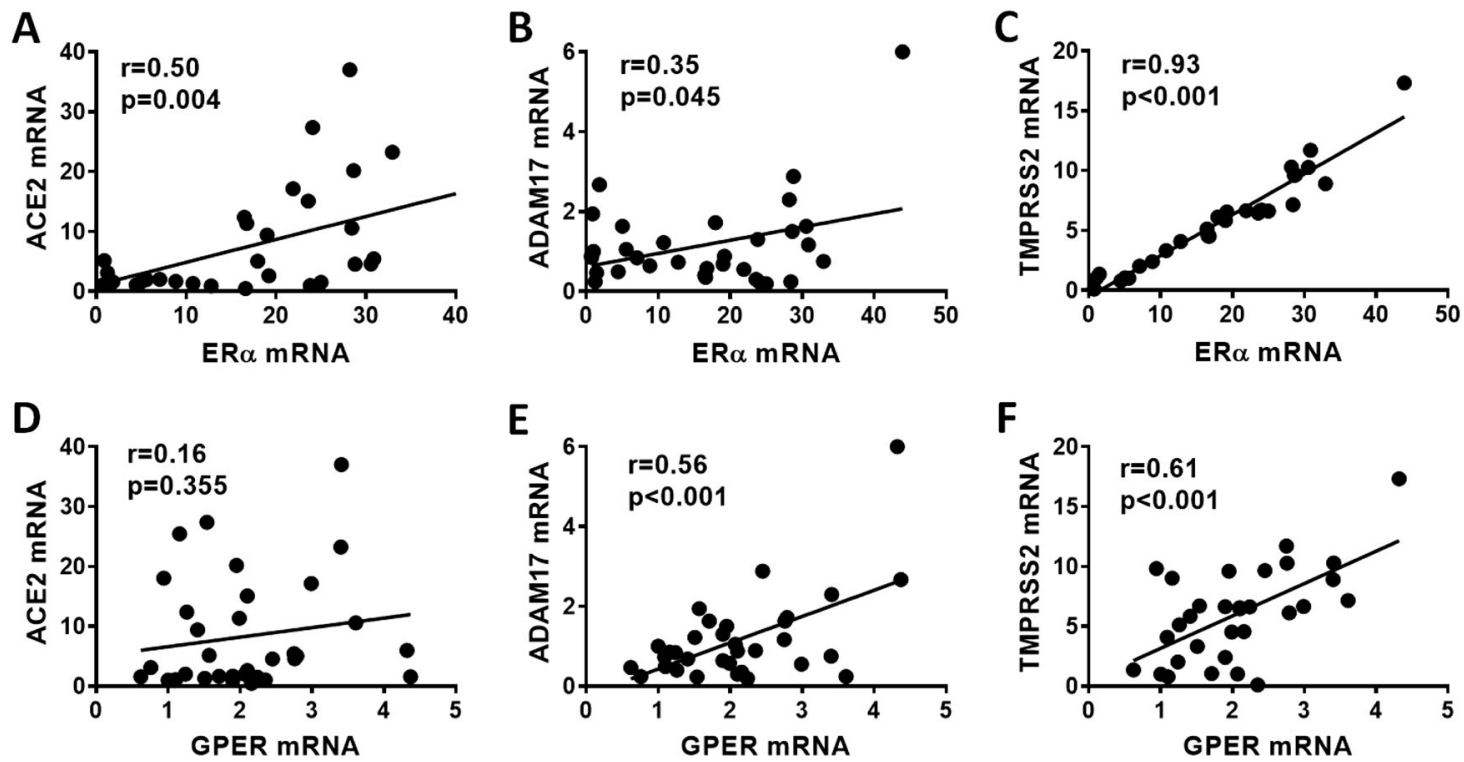

Fig. 1 Correlations among mRNA levels of estrogen receptors (ERs) and ACE2/ADAM17/TMPRSS2 in the human atrium. Right atrial appendage tissues were collected from 34 patients undergoing heart surgery for the correction of valvular heart disease, resistant atrial fibrillation or ischemic heart disease (mean age $65 \pm 7$ years of age,
$79 \%$ men). The study was approved by the Wake Forest University Health Sciences (IRB 22619). mRNA levels in the atrium were determined by real-time PCR and corrected by the housekeeping gene GAPDH. Correlations between mRNA levels were determined using GraphPad Prism 8 software elevated ACE2 expression might facilitate viral entrance. In view of this dual role of tissue ACE2, the sexual dimorphic pattern of SARS-CoV-2 infection severity might involve multiple mechanisms, including differences in immune system responses between women and men. Further investigations of the exact roles of ACE2 and its interaction with estrogen in COVID-19 patients with CVDs are needed to address the potential benefit of sex-specific therapeutic interventions to protect against disease progression.

In addition, we discovered that the mRNA levels of both $\mathrm{ER} \alpha$ (Fig. 1B, C) and GPER (Fig. 1E, F), but not ER $\beta$ (data not shown), correlated positively with ADAM17 and TMPRSS2 in human right atrial appendage tissues. Indeed, the TMPRSS2 gene transcript showed the highest level of association with $\mathrm{ER} \alpha$, as illustrated in panels $\mathrm{C}$ and $\mathrm{F}$ of the figure. To our knowledge, this is the first report demonstrating strong associations between estrogen receptors and the expression levels of ADAM-17 and TMPRSS2 in human cardiac tissue, providing new insight into the relationship between estrogen and the heart.

These results from atrial appendage tissue suggest that estrogen, primarily through its receptors ER $\alpha$ and GPER, affects cardiac ACE2 levels and activity by regulating ACE2 shedding via ADAM-17 and TMPRSS2. Interestingly, the linear correlation of ACE2 gene transcripts with ER $\alpha$ and GPER mRNAs appears to be associated with higher values of ACE2 gene expression. This new finding may assist in explaining the discordant results reported in the literature regarding the impact of SARS-CoV-2 infection on plasma ACE2 activity [9]. We realize the limitations of our reported data. Due to a small sample size, we used tissue from patients with a variety of cardiac pathologies and treatments, which might have influenced the expression levels of estrogen receptors, ACE2, ADAM17, and TMPRSS2. Future studies focused on specific cardiac conditions will advance our understanding of the potential relationships among estrogen receptors and ACE2, ADAM-17, and TMPRSS2. Moreover, tissue samples from both sexes were included in this study. Although estrogen receptors are similarly expressed in the heart of males and females and estradiol protects the heart against various stresses in both male and female animals [6, 10], future studies with sex separation are needed to elucidate how estrogen regulates cardiac ACE2 in both sexes. As only mRNA levels were determined in this study due to the small amount of tissue obtained from each patient, protein levels and enzymatic activities of ACE2, ADAM-17, and TMPRSS2 should be further examined to elucidate how activation of ER $\alpha$ and GPER affects ACE2 shedding and therefore participates in CVD and COVID-19. Nevertheless, the novel demonstration of associations among cardiac ACE2, ADAM-17, and TMPRSS2 gene transcripts with ER $\alpha$ and GPER mRNA levels reveals for the first time a functional modulatory mechanism of estrogen receptors for the structural elements that may regulate ACE2.

Funding This research was supported by the National Institutes of Health (HL-051952 to CMF, AG042758 and AG033727 to LG). 


\section{Compliance with ethical standards}

Conflict of interest The authors declare that they have no conflict of interest.

Publisher's note Springer Nature remains neutral with regard to jurisdictional claims in published maps and institutional affiliations.

\section{References}

1. Engberding N, Wenger NK. Management of hypertension in women. Hypertens Res. 2012;35:251-60.

2. Zipeto D, Palmeira JDF, Argañaraz GA, Argañaraz ER. ACE2/ ADAM17/TMPRSS2 interplay may be the main risk factor for COVID-19. Front Immunol. 2020;11:576745.

3. Groban L, Wang H, Sun X, Ahmad S, Ferrario CM. Is sex a determinant of COVID-19 infection? truth or myth? Curr Hypertens Rep. 2020;22:62.

4. Shibata S, Arima H, Asayama K, Hoshide S, Ichihara A, Ishimitsu $\mathrm{T}$, et al. Hypertension and related diseases in the era of
COVID-19: a report from the Japanese Society of Hypertension Task Force on COVID-19. Hypertens Res. 2020;43:1028-46.

5. Wang Z, Wang Y, Zhang J, Hu Q, Zhi F, Zhang S, et al. Significance of the TMPRSS2:ERG gene fusion in prostate cancer. Mol Med Rep. 2017;16:5450-8.

6. Hutson DD, Gurrala R, Ogola BO, Zimmerman MA, Mostany R, Satou R, et al. Estrogen receptor profiles across tissues from male and female Rattus norvegicus. Biol Sex Differ. 2019;10:4.

7. Bukowska A, Spiller L, Wolke C, Lendeckel U, Weinert S, Hoffmann J, et al. Protective regulation of the ACE2/ACE gene expression by estrogen in human atrial tissue from elderly men. Exp Biol Med. 2017;242:1412-23.

8. Ferrario CM, Ahmad S, Groban L. Mechanisms by which angiotensin-receptor blockers increase ACE2 levels. Nat Rev Cardiol. 2020;17:378.

9. Li X, Liu Y, Song J, Zhong J. Increased plasma ACE2 concentration does not mean increased risk of SARS-CoV-2 infection and increased fatality rate of COVID-19. Acta Pharm Sin B. 2020;10:2010-4.

10. Imanishi T, Kobayashi K, Hano T, Nishio I. Effect of estrogen on differentiation and senescence in endothelial progenitor cells derived from bone marrow in spontaneously hypertensive rats. Hypertens Res. 2005;28:763-72. 